\title{
Molecular Docking of molecules isolated from Malvaceae family focusing in the development of antithrombotic agents
}

\author{
Wallison S. Dias* (IC), Yanna C. F. Teles (PQ), Sidney R. Santana (PQ); \\ Depart. of Chemistry and Physics, Center of Agrarian Center, Federal University of Paraíba (UFPB). \\ e-mail: santanasidney@cca.ufpb.br
}

Keywords: Molecular Docking, Antithrombotic Agents, Smina.

\section{Introduction}

The Malvaceae family is estimated to contain 243 genera with 4225 species. From that, sulphated flavonoids was isolated, tested and showed some anti thrombosis activity[1].

Due the diversity of kind molecules usually presented in medicinal chemistry, molecular docking has been presented as a first step evaluation needed to the rationalization development of drug-like compounds[2].

In this work, we evaluated two different scoring functions implemented in the same program to describe affinities of that kind of flavonoids molecules into some thrombosis biological targets.

\section{Methodology}

All docking experiments were conducted using Smina programs[3], testing two of its scoring functions[4]: Vinardo e DK. The docking grid was centered on the cocrystallized ligand with a size of $40 \AA \times 40 \AA \times 40 \AA$ and a grid spacing of $1 \AA$. The exhaustiveness was equal 30 . The By default, the docking was terminated when the maximum energy difference between the best scored pose and the worst one was $3 \mathrm{kcal} /$ mol.

Five biological target was chosen in Protein Databank[5] by its code 1HSG, 2B7D, 2JEW, 4MHA, 4TY6. The results was established by comparisons between affinity energies of commercial drug co-crystallized and the trial molecules named M01, M02, M03, M04, M05.

\section{Results}

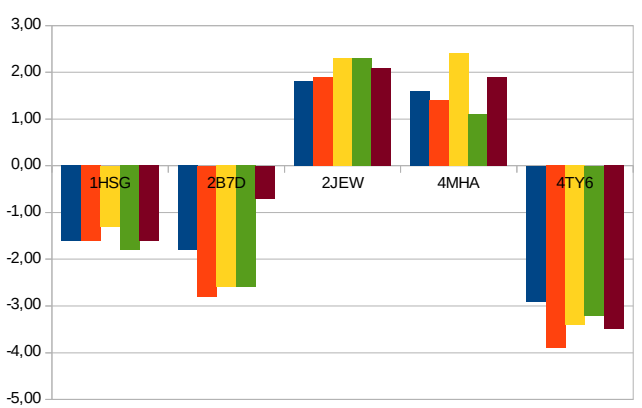

Figure 1: Vinardo Scoring Affinity Energy Results

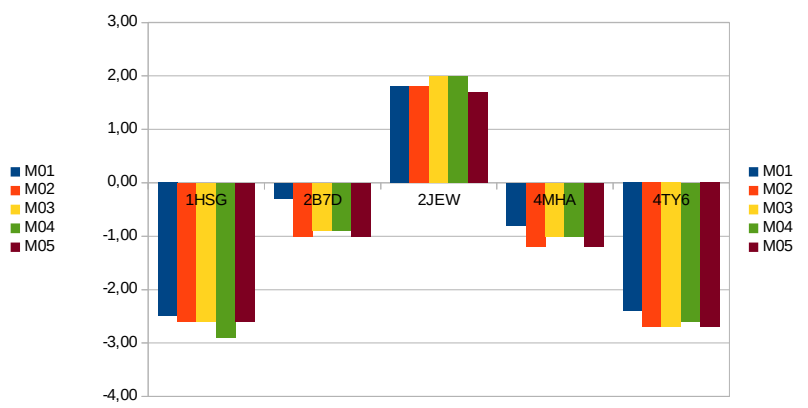

Figure 2: Dkoes Scoring Affinity Energy Results 
The Figures 1 and 2 showed us the affinity energies differences between the commercial drugs versus the drug-like molecules (M01,M02, M03, M04, M05) to each biological target, largest is better.

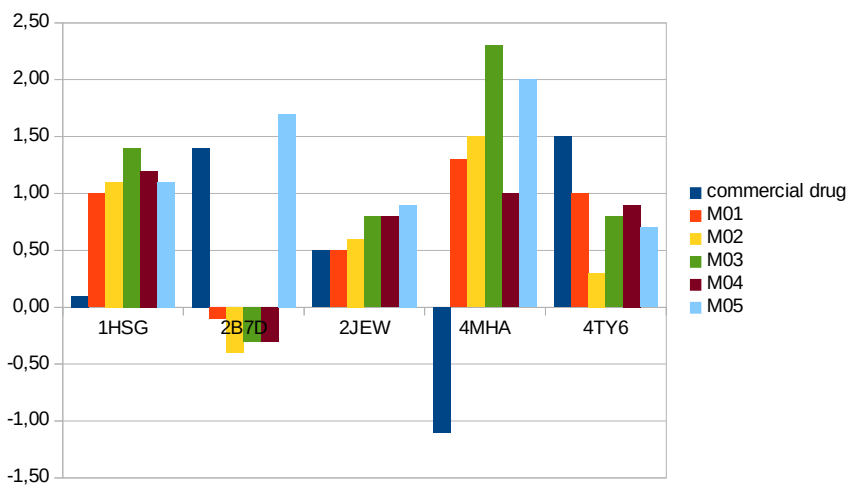

Figure 3: Affinity Energy Differences between DK and Vinardo Scoring functions.

The Figure 3 showed us affinity Energy Differences between DK and Vinardo scoring functions, largest is better.

\section{Discussion}

The DK scoring function presented better results than Vinardo one.

That scoring functions employed in this molecular docking work produced good results for at least one target, $2 \mathrm{JEW}$, noted the same trends for all drug-like molecules.

\section{Conclusions}

The smina program help us to establish reasonable affinity energies and the DK scoring functions seem to be better employed to describe thrombosis biological target, yelding at good candidates to new future drugs against this disease.

\section{Acknowledgments}

\section{CAPES, CNPq, UFPB, Cenapad/SP}

\section{References}

[1] Y. C. F. Teles; el. al.; Molecules 2015, 20, 20161-20172

[2] M. Gore, U. B. Jagtap; Computational Drug Discovery and Design, Methods in Molecular Biology, vol. 1762, Chapter 9, pp. 145-178, Springer, 2018.

[3] Smina Programs, https://sourceforge.net/projects/smina/

[4] R. Quiroga; et. al.; PLoS ONE 2016, 11(5): e0155183

[5] Protein DataBank, https://www.rcsb.org 